\title{
Genes del receptor variable beta de células T en células circulantes de pacientes con lupus eritematoso generalizado y sus familiares sanos
}

\author{
Juan Jakez-Ocampo', Carmen María Paulín-Vera1, Liliana Rivadeneyra-Espinoza², Diana Gómez-Martín', \\ Eduardo Carrillo-Maravilla³, Guadalupe Lima1, María Inés Vargas-Rojas ${ }^{1}$, Beatriz Pérez-Romano², \\ Gabriella Calva-Cevenini2, Mario García-Carrasco ${ }^{4}$, Alejandro Ruiz-Argüelles ${ }^{2}$ y Luis Llorente ${ }^{1}$ \\ 'Departamento de Inmunología y Reumatología, Instituto Nacional de Ciencias Médicas y Nutrición Salvador Zubirán, Ciudad de México; \\ ${ }^{2}$ Laboratorios Clínicos de Puebla, Puebla, Pue.; ${ }^{3}$ Departamento de Medicina Interna, Instituto Nacional de Ciencias Médicas y Nutrición Salvador \\ Zubirán, Ciudad de México; ${ }^{4}$ Escuela de Medicina, Benemérita Universidad Autónoma de Puebla, Puebla, Pue. México
}

\begin{abstract}
Resumen
Objetivo: Se investigó la proporción de la expresión génica del receptor variable beta de células T (VB TCR) en linfocitos periféricos CD3+ en pacientes con lupus eritematoso generalizado (LEG) familiar y no familiar. Método: El repertorio de VB TCR se estudió en 14 familias que presentaban más de un miembro con LEG. El uso de VB TCR en pacientes con LEG $(n=27)$ se comparó con el de los miembros sanos de estas familias $(n=47)$, con 37 pacientes con $L E G$ esporádico y con 15 controles sanos. La expresión del repertorio de VB TCR se estudió por citometría de flujo multiparamétrica utilizando un arreglo de 24 diferentes anticuerpos monoclonales específicos de genes familiares para VB TCR. Resultados: Se encontró el mismo perfil de expresión en las comparaciones entre los casos de LEG esporádico y familiar, así como en los consanguíneos sanos de las familias multicasos, que incluía una expresión incrementada de $V \beta$ 5.2, VB 11 y VB 16, y una menor expresión de $V \beta 3$, VB4, VB 7.1 y VB 7. De manera interesante, solo VB 17 se expresó de modo diferente entre casos familiares y esporádicos de LEG. Igualmente, la expresión incrementada de VB 9 fue el distintivo entre los casos de LEG familiar (casos y consanguíneos sanos) y los controles sanos. Conclusiones: Estos resultados refuerzan la noción de que el perfil final del repertorio VB TCR observado en LEG familiar y no familiar parece surgir de la interacción de factores genéticos, ambientales e inmunorreguladores, además de que pueden explicar las alteraciones inmunitarias que se observan en los consanguíneos sanos de pacientes con LEG.
\end{abstract}

PALABRAS CLAVE: Repertorio V TCR. Lupus eritematoso generalizado. Lupus eritematoso esporádido. Lupus eritematoso familiar.
Abstract
Objective: We investigated the proportion of $V \beta T$ cell receptor (TCR) gene expression in peripheral CD3+ lymphocytes in familial and non-familial systemic lupus erythematosus (SLE) patients. Method: The VB TCR repertoire was studied in 14 families in which several members had SLE. The VB TCR usage in SLE patients $(n=27)$ was compared with that in healthy members of these multiplex families $(n=47)$, in 37 sporadic SLE patients who had no relatives with SLE, and in 15 healthy unrelated controls. VB TCR repertoire expression was studied by multiparameter flow cytometry with the use of an array of 24 different VB TCR gene family-specific monoclonal antibodies. Results: We found the same VB TCR expression profile in the

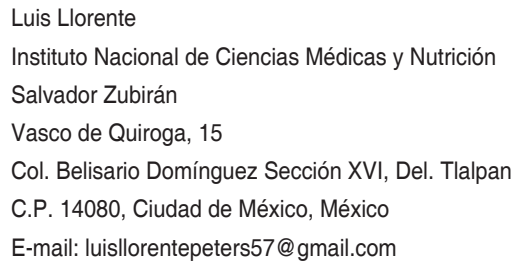
Gac Med Mex. 2018;154:74-79
Contents available at PubMed www.gacetamedicademexico.com 
comparisons between sporadic SLE and familial SLE cases and healthy relatives, which included increased expression of VB 5.2, $V \beta 11$ and $V \beta 16$, and lower expression of $V \beta 3, V B 4, V \beta 7.1$ and $V \beta$ 17. Interestingly, solely $V \beta 17$ was differentially expressed among sporadic and familial SLE. Also, increased expression of VB 9 was the hallmark among familial SLE (cases and healthy relatives) in comparison to controls. Conclusion: These results highlight the notion that the final profile of the $V B$ TCR repertoire seen in familial and non-familial SLE seems to arise from the interaction of genetic, environmental, and immunoregulatory factors. Furthermore, it may contribute to the immunologic abnormalities affecting relatives of SLE patients.

KEY WORDS: VB T cell receptor repertoire. Systemic lupus erythematosus. Sporadic lupus erythematosus. Familiar systemic lupus erythematosus.

\section{Introducción}

Las células T desempeñan un papel importante en la patogénesis del lupus eritematoso generalizado $(\mathrm{LEG})^{1}$. Una manera de distinguir clonas relevantes a la enfermedad es a través del estudio del receptor de células $T$ (TCR), mediante el cual las células $T$ reconocen antígenos presentados en interacción con el complejo principal de histocompatibilidad ${ }^{2}$. Se cree que la cadena beta del TCR es una de las estructuras más importantes para la especificidad antigénica ${ }^{3}$. Los genes que codifican la región variable de la cadena beta pueden dividirse en 24 familias que comparten secuencias similares ${ }^{4}$, y por lo tanto pueden ser de utilidad en la identificación de subgrupos de células $T$ activas en enfermedades autoinmunes, en las que se cree que un número limitado de autoantígenos son la causa del inicio y de la progresión de la enfermedad. Sin embargo, la información respecto al uso de los genes del receptor variable beta de células T (V $\beta$ TCR) en enfermedades autoinmunes es muy escasa, controversial y se ha enfocado principalmente en el análisis de células T tisulares en el sitio de la lesión ${ }^{5-7}$. En pacientes con síndrome de Sjögren primario y lesiones linfoproliferativas se han detectado cifras elevadas de células $T$ que expresan moléculas codificadas por la familia $\mathrm{V} \beta 2^{8}$, y se ha propuesto el papel de ciertos autoantígenos locales como los causantes de la expansión clonal ${ }^{9}$. Tanto en pacientes con nefritis lúpica como en pacientes con lupus cutáneo se ha demostrado que las células $T$ que infiltran la lesión renal y la piel exhiben una proporción mayor de células portadoras de moléculas TCR codificadas por la familia $\mathrm{V} \beta 8, \mathrm{~V} \beta 20$ y $\mathrm{V} \beta 14^{10,11}$, y de nuevo no se ha podido descartar la expansión in situ de las clonas de células $T$ que poseen este rearreglo particular de los genes de V $\beta$.

En este estudio se describen las proporciones de células $T$ periféricas que muestran en su receptor moléculas codificadas por las 24 familias de genes que codifican para el receptor $\mathrm{V} \beta$ en pacientes con LEG familiar y no familiar o esporádico, así como en sus familiares consanguíneos de primer y segundo grado. Esto último debido a que una desregulación inmunitaria similar a aquella observada en los pacientes con LEG ha sido descrita en estas personas ${ }^{12-15}$. Este acercamiento permite tener control sobre los efectos genéticos que pueden influir en la susceptibilidad a la enfermedad, lo cual podría ser un patrón de respuesta específico a la herencia de un grupo de genes que contribuyan al LEG.

\section{Método}

El estudio incluyó 27 pacientes con LEG que eran miembros de 14 familias con al menos dos miembros con LEG, 47 familiares consanguíneos sanos miembros de estas 14 familias, 37 pacientes con LEG esporádico y 15 controles sanos sin relación con estas familias. Todos los pacientes con LEG cumplían los criterios revisados del American College of Rheumatology ${ }^{16}$. Catorce pacientes de las familias con casos múltiples y 28 pacientes con LEG esporádico recibían tratamiento con corticoesteroides con o sin fármacos inmunosupresores. En las condiciones experimentales utilizadas, el tratamiento no tenía ningún efecto sobre el uso de las familias de V $\beta$ TCR.

En las familias con casos múltiples de LEG, los familiares de primer grado y segundo grado fueron también estudiados, además de los pacientes. Todos los sujetos incluidos en el estudio son considerados mestizos mexicanos, es decir, que nacieron en México al igual que sus últimas dos generaciones. Todos los participantes en el estudio recibieron información sobre los objetivos y los métodos del estudio, y dieron su consentimiento informado.

El análisis del repertorio V $\beta$ TCR fue realizado por citometría de flujo. Se tomaron muestras de sangre periférica de todos los participantes en tubos con EDTA. Se incubaron $50 \mu \mathrm{l}$ de sangre con $5 \mu \mathrm{l}$ de cada 
mezcla de tres anticuerpos monoclonales específicos para la familia $\mathrm{V} \beta$ del kit del repertorio de $\mathrm{V} \beta$ TCR (Beckman Coulter Immunotech, Marseille, France), y $5 \mu \mathrm{l}$ de anti-CD3 conjugado con aloficocianina (Immunotech). Después de incubación en hielo por $15 \mathrm{mi}$ nutos, y los ocho tubos de cada sujeto fueron hemolisados y fijados con la ayuda de un sistema automatizado (ImmunoPrep® Beckman Coulter, Miami. FLA, USA). Las células se analizaron en un citómetro de flujo EPICS Elite ESP (Beckman Coulter) o con Cytomics FC500 (Beckman Coulter). En ambos citómetros el isiotiocianato de fluoresceína y la ficoeritrina se excitaron con un láser de argón enfriado por aire a $488 \mathrm{~nm}$, mientras que la aloficocianina fue excitada con un láser de helio-neón a $633 \mathrm{~nm}$. Las emisiones de los tres fluorocromos fueron capturadas a 525, 575 y $675 \mathrm{~nm}$, respectivamente. Las proporciones de las células que expresaban cada uno de los tres genes $\mathrm{V} \beta$ en cada mezcla (una conjugada con isiotiocianato de fluoresceína, otra con ficoeritrina y otra con ambos fluorocromos) se determinaron en células CD3+. Un total de 10,000 células CD3+ se cuantificaron en cada experimento ${ }^{17}$.

La expresión de cada uno de los genes de V $\beta$ TCR se describió como media \pm desviación estándar. La comparación de la expresión de los genes de V $\beta$ TCR entre grupos (LEG esporádico, LEG familiar, familiares sanos y controles) se hizo por medio de ANOVA de una vía y corrección de Dunnett's para múltiples comparaciones. La asociación de la actividad clínica de los pacientes y los cambios en la expresión de V $\beta$ TCR se analizó por la prueba no paramétrica exacta de Fisher. Se consideró una diferencia significativa con un valor de $p<0.05$.

\section{Resultados}

El repertorio de V $\beta$ TCR se estudió en 14 familias con casos múltiples de LEG. El empleo de V $\beta$ TCR en pacientes con LEG $(n=27)$ se comparó con el de los miembros sanos de la misma familia $(n=47)$, con 37 pacientes con LEG esporádico y con 15 controles sanos no relacionados con ninguno de los anteriores. El análisis estadístico no mostró diferencias en relación con el sexo y la edad entre pacientes y controles. En el caso de los pacientes, tampoco se encontraron diferencias respecto al tiempo de evolución de la enfermedad, la actividad clínica determinada con SLEDAl y el tratamiento.

La tabla 1 muestra los valores de la media y la desviación estándar de las proporciones de células T
Tabla 1. Proporciones de células CD3+ que expresan diferentes genes V $\beta$ TCR en los diferentes grupos de estudio

\begin{tabular}{|c|c|c|c|c|c|c|c|c|}
\hline & \multicolumn{2}{|c|}{ Controles } & \multicolumn{2}{|c|}{$\begin{array}{c}\text { LEG } \\
\text { esporádico }\end{array}$} & \multicolumn{2}{|c|}{ LEG familiar } & \multicolumn{2}{|c|}{$\begin{array}{c}\text { Familiares } \\
\text { sanos }\end{array}$} \\
\hline & Media & DE & Media & DE & Media & DE & Media & DE \\
\hline$V \beta 1$ & 2.530 & 0.736 & 3.741 & 1.508 & 3.523 & 1.073 & 3.471 & 1,170 \\
\hline$\vee \beta 2$ & 5.941 & 0,8 & 6.695 & 2.285 & 6.803 & 2.123 & 6.961 & 2.182 \\
\hline$V \beta 3$ & 6.530 & 2.792 & 2.381 & 1.772 & 4.363 & 3.001 & 4.451 & 3.161 \\
\hline$V \beta 4$ & 2.951 & 2.899 & 1.315 & 1.243 & 2.339 & 1.273 & 2,352 & 0.955 \\
\hline$V \beta 5.1$ & 4.253 & 1.281 & 4.088 & 2.092 & 5.173 & 1.625 & 5.180 & 1.808 \\
\hline$V \beta 5.2$ & 1.719 & 0.648 & 1.997 & 0.973 & 1.068 & 0.454 & 1.164 & 0.590 \\
\hline V $\beta 5.3$ & 1.261 & 0.371 & 1.565 & 1.336 & 1.209 & 0.455 & 1.514 & 1.769 \\
\hline$V \beta 7.1$ & 2.578 & 0.626 & 1.693 & 1.050 & 3.074 & 1.754 & 2.830 & 2.048 \\
\hline$V \beta 7.2$ & 2.286 & 1,313 & 1.512 & 0.989 & 0.922 & 0.942 & 1.213 & 1.109 \\
\hline$V \beta 8$ & 3.881 & 0.931 & 4.424 & 2.762 & 4.871 & 1.451 & 4.784 & 1.256 \\
\hline$\vee \beta 9$ & 1.938 & 0,438 & 3.964 & 4.585 & 4.909 & 2.833 & 4.496 & 2.976 \\
\hline $\mathrm{V} \beta 11$ & 2.150 & 1.638 & 2.083 & 1.426 & 0.684 & 0.326 & 0.841 & 0.497 \\
\hline V $\beta 12$ & 1.900 & 0.952 & 2.201 & 2.827 & 1,553 & 0.597 & 1.778 & 0868 \\
\hline$V \beta 13.1$ & 3.129 & 0.653 & 3.713 & 1.183 & 4.044 & 1.369 & 4.371 & 2.842 \\
\hline V $\beta 13.2$ & 3.341 & 2.816 & 3.133 & 4.442 & 2.890 & 2.181 & 2.705 & 1.162 \\
\hline$V \beta 13.6$ & 1.869 & 0.987 & 1535 & 0.678 & 1.759 & 0.522 & 1.521 & 0.592 \\
\hline$V \beta 14$ & 3.030 & 0.862 & 2.606 & 3.465 & 3.600 & 2.301 & 2.763 & 1.159 \\
\hline$V \beta 16$ & 1.421 & 0.875 & 2.484 & 2.947 & 0.906 & 0.364 & 1.039 & 0.539 \\
\hline$V \beta 17$ & 4.006 & 1.182 & 3.095 & 2.143 & 5.764 & 1.842 & 4.647 & 1.114 \\
\hline V $\beta 18$ & 2.263 & 1478 & 2.051 & 2.721 & 1.117 & 1.591 & 0.815 & 0,716 \\
\hline V $\beta 20$ & 2.439 & 1.095 & 2.483 & 1.261 & 2.164 & 1.102 & 2.190 & 1.031 \\
\hline V $\beta 21.3$ & 2.094 & 0.99 & 2.596 & 2.002 & 2.628 & 1.314 & 2.444 & 1,395 \\
\hline$V \beta 22$ & 3.509 & 0.637 & 3.462 & 1.708 & 4.325 & 1.251 & 4.298 & 1.366 \\
\hline$V \beta 23$ & 2.743 & 1.981 & 1.334 & 1.629 & 0.821 & 0.441 & 0.917 & 0.759 \\
\hline
\end{tabular}

CD3+ expresando cada uno de los genes de las 24 familias $\mathrm{V} \beta \mathrm{TCR}$.

La figura 1 representa la media de las proporciones de los genes de la familia $V \beta$ TCR en pacientes con LEG esporádico, LEG familiar y sus familiares sanos, expresados como la desviación de los valores de la media obtenidos en el grupo control. Esta desviación fue calculada por la fórmula:

$\%$ desviación $=[$ (valor de la media del grupo de estudio/valor de la media del grupo control) - 1] × 100

la cual es un estimado del incremento o la disminución relativos de la expresión de estas familias de genes en los diferentes grupos. 


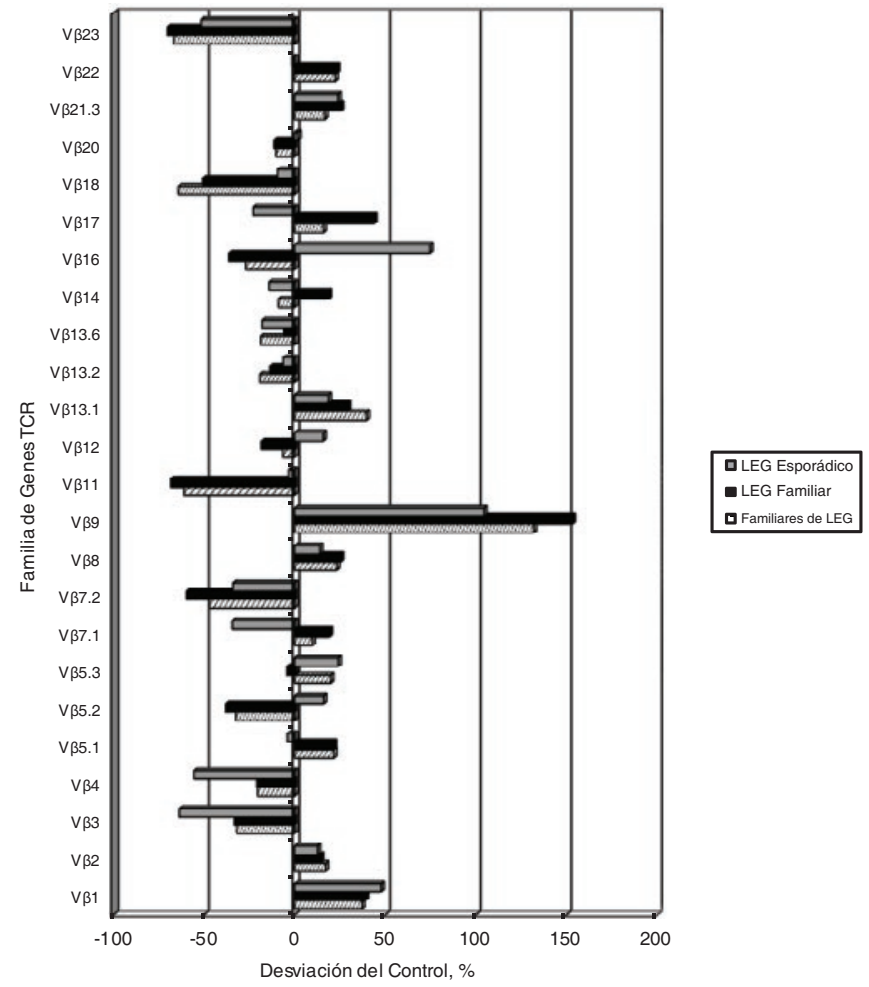

Figura 1. Desviación relativa de los valores medios de las proporciones de células T que expresan cada uno de los 24 genes de la familia VB TCR en pacientes con lupus eritematoso generalizado (LEG) esporádico, familiar y sus familiares sanos, comparados con el valor medio del grupo control.

Se encontró el mismo perfil de expresión de genes $\mathrm{V} \beta$ TCR en las comparaciones entre LEG esporádico, casos familiares y familiares sanos, los cuales incluyeron una expresión aumentada de $\mathrm{V} \beta 5.2, \mathrm{~V} \beta$ 11 y $\vee \beta 16$, así como una expresión disminuida de $V \beta$ $3, \vee \beta 4, V \beta 7.1$ y $\vee \beta 17$.

De manera interesante, únicamente $V \beta 17$ fue expresado de modo diferente en el grupo de LEG esporádico y LEG familiar, con una expresión aumentada entre los casos de LEG familiar cuando se comparó con los familiares sanos y los controles, así como una expresión disminuida en LEG esporádico en comparación con LEG familiar (casos y familiares sanos). Asimismo, se observó de manera distintiva una expresión aumentada de la familia V $\beta 9$ entre los casos de LEG familiar (pacientes y familiares sanos) en comparación con los controles (Tabla 2).

\section{Discusión}

Nuestros resultados muestran que el porcentaje del empleo del dominio $V \beta$ en linfocitos T CD3+ en sujetos sanos fue similar a lo reportado previamente en la literatura utilizando la misma metodología ${ }^{18}$. Así, con este abordaje experimental se encontró el mismo perfil de expresión en pacientes con LEG esporádico en comparación con los casos de LEG familiar y sus familiares sanos, que se caracterizó por la expresión al alza de $\vee \beta$ 5.2, $\vee \beta 11$ y $\vee \beta 16$, así como una expresión a la baja de $\mathrm{V} \beta 3, \mathrm{~V} \beta 4, \mathrm{~V} \beta$ 7.1 y $\mathrm{V} \beta$ 17. Este perfil de expresión sugiere diferencias en el empleo de genes V $\beta$ TCR entre los casos de LEG esporádico y familiar. Estas diferencias se encontraron también cuando se compararon con los familiares sanos de las familias multicaso, pero no en los controles sanos no relacionados con ellas.

Este sesgo y la expansión de las células $T$ en sangre periférica de pacientes con LEG puede ser resultado de la activación y la proliferación de clonas relevantes a la enfermedad. De hecho, se ha reportado que en las células $T$ de pacientes con LEG la región determinante de complementación 3 (CDR3) muestra un empleo restringido de genes $V \alpha$ y $V \beta$, lo que puede sugerir la expansión clonal de células $T$ en relación con un número limitado de epítopos antigénicos ${ }^{19,20}$. En concordancia con lo anterior, un sesgo en el uso del TCR ha sido relacionado con autoinmunidad, como en el caso del modelo múrido de encefalomielitis autoinmunitaria experimental ${ }^{21}$. 
Tabla 2. Diferencias significativas en la expresión de genes V $\beta$ TCR entre pacientes con lupus eritematoso generalizado esporádico, pacientes con lupus eritematoso generalizado familiar, familiares sanos y controles

\begin{tabular}{|c|c|c|c|c|c|c|c|c|c|}
\hline & $V \beta 1$ & $V \beta 3$ & V $\beta 4$ & $V \beta 5.2$ & $V \beta 7.1$ & $V \beta 9$ & V $\beta 11$ & V $\beta 16$ & V $\beta 17$ \\
\hline LEG esporádico vs. LEG familiar & & $\downarrow$ & $\downarrow$ & $\uparrow$ & $\downarrow$ & & $\uparrow$ & $\uparrow$ & $\downarrow$ \\
\hline LEG familiar vs. familiares sanos & & & & & & & & & $\uparrow$ \\
\hline LEG esporádico vs. familiares sanos & & $\downarrow$ & $\downarrow$ & $\uparrow$ & $\downarrow$ & & $\uparrow$ & $\uparrow$ & $\downarrow$ \\
\hline LEG esporádico vs. controles & $\uparrow$ & $\downarrow$ & & & $\downarrow$ & & & & \\
\hline LEG familiar vs. controles & $\uparrow$ & & & & & $\uparrow$ & & & $\uparrow$ \\
\hline Familiares sanos vs. controles & $\uparrow$ & & & & & $\uparrow$ & & & \\
\hline
\end{tabular}

$\uparrow \downarrow$ : Significancia $(p<0.05)$ respecto a aumento/disminución en la proporción de células CD3+ expresando cada uno de los diferentes genes V $\beta$ TCR. Las celdas en blanco representan ausencia de significancia estadística. Las familias de genes en que las diferencias no fueron significativas no se muestran en la tabla.

LEG: lupus eritematoso generalizado.

Estos resultados sugieren que tales diferencias en el empleo de TCR V $\beta$ podrían también estar involucradas en las anormalidades inmunitarias previamente descritas en los familiares de pacientes con LEG ${ }^{12-}$ ${ }^{15}$, y podrían representar un factor de susceptibilidad al desarrollo de la enfermedad, aun cuando esto necesariamente requiere la presencia de otros factores genéticos o ambientales. Esto pudiera representar la diferencia entre autoinmunidad y enfermedad autoinmunitaria. Por lo tanto, el concepto de mosaico de autoinmunidad adquiere sentido ${ }^{22}$.

En concordancia con un estudio previo ${ }^{19}$ en el cual se utilizó un acercamiento metodológico diferente, denominado tipificación espectral, también se encontró un empleo predominante de los genes $V \beta 11$ y $V \beta 16$ entre los pacientes con LEG, tanto esporádicos como familiares. Sin embargo, el artículo antes mencionado no incluyó un análisis con familias de casos múltiples, como el realizado en este trabajo.

Notablemente, V $\beta 17$ fue el único gen TCR expresado de forma diferente entre los casos de LEG esporádico y familiar. En los casos esporádicos, su expresión es baja en comparación con los casos familiares y sus familiares sanos. Nuestros resultados sugieren que las anormalidades en el empleo de V $\beta$ 17 pueden ser parte de defectos inmunorreguladores en el LEG, y su expresión puede ayudar a identificar susceptibilidad genética entre familiares sanos. La expresión diferente de este gen entre casos esporádicos y familiares de LEG puede tener implicaciones funcionales relacionadas con la especificidad antigénica de la respuesta inmunitaria, como se ha mostrado en el caso del empleo de V $\beta 14$, el cual se relaciona con la autoinmunidad hacia histonas $y$ nucleosomas ${ }^{23}$. De hecho, Desai-Mehta A, et al. ${ }^{24}$ reportaron un aumento de los títulos de anticuerpos
anti-DNA de doble cadena inducidos por una estirpe de células Th en un paciente con lupus con empleo predominante de V $\beta$ 17. Sin embargo, esta cuestión requiere mayor estudio para aclarar su implicación patogénica.

También la expresión incrementada de $V \beta 9$ fue el rasgo característico en el LEG familiar (casos y familiares sanos) en comparación con los controles sanos no relacionados, por lo que su sobreexpresión puede ser un indicador del antecedente genético, enfatizando el requerimiento de otros factores genéticos, así como ambientales, para que en conjunto se relacionen con la expresión clínica de la enfermedad.

Finalmente, no se encontró asociación con la actividad de la enfermedad, como ha sido reportado por otros autores ${ }^{19,25}$. Sin embargo, el análisis de esta asociación no se encontraba dentro los objetivos de nuestro estudio y requiere mayor investigación.

Hasta donde sabemos, este es el primer estudio sobre el empleo de genes V $\beta$ TCR que compara grupos de LEG esporádico y familiar, así como a sus familiares sanos. Nuestros resultados sugieren que el empleo de algunos genes de TCR V $\beta$ en estos grupos de pacientes es diferente. Ciertamente, una limitante del trabajo está dada por un tamaño de muestra menor que el deseable, pero las familias de casos múltiples para LEG son limitadas tanto en número como en disponibilidad.

Si bien otros acercamientos metodológicos, como la tipificación espectral, pueden ser más útiles para detallar las características moleculares de estos genes TCR V $\beta$, nuestro abordaje podría considerarse como el paso inicial para profundizar en el análisis de aquellos genes que muestran una expresión diferente entre casos esporádicos y familiares de LEG, como lo es el V $\beta 17$. 


\section{Bibliografía}

1. Kyttaris VC, Katsiari CG, Juang YT, et al. New insights into the pathogenesis of systemic lupus erythematosus. Curr Rheumatol Rep. 2005;7:469-75.

2. Goldrath AW, Bevan MJ. Selecting and maintaining a diverse T-cell repertoire. Nature. 1999;402:255-62.

3. Lone YC, Ojcius DM, Bellio M, et al. Major contribution of the beta chain to the antigen specificity of a $\mathrm{T}$ cell receptor. Crit Rev Acad Sci Paris. 1994:317:645-51.

4. Wei S, Charmley $P$, Robinson MA, et al. The extent of the human germline $\mathrm{T}$-cell receptor $\mathrm{V} \otimes$ gene segment repertoire. Immunogenetics. 1994:40:27-36.

5. Pivetta B, De Vita S, Ferraccioli G, et al. T cell receptor repertoire in B cell lymphoproliferative lesions in primary Sjögren's syndrome. J Rheumatol. 1999:26:1101-9.

6. Kelsen J, Agnholt J, Hoffmann HJ, et al. Increased expression of TCR V $\beta 5.1$ and 8 in mucosal T-cell lines cultured from patients with Crohn disease. Scand J Gastroenterol. 2004;39:238-45.

7. Yanagi K, Haneji N, Ishimaru N, et al. Analysis of $\mathrm{T}$ cell receptor $\mathrm{V}$ beta usage in the autoimmune sialadenitis of non-obese diabetic (NOD) mice. Clin Exp Immunol. 1997;109:555-61.

8. Manavalan SJ, Valiando JR, Reeves WH, et al. Genomic absence of the gene encoding $T$ cell receptor $V$ beta 7.2 is linked to the presence of autoantibodies in Sjögren's syndrome. Arthritis Rheum. 2004;50: 187-98.

9. Hauke G, Rude B, Hollmann A, et al. Immunoglobulin and T-cell receptor gene rearrangements in patients with autoimmune diseases. Immun Infeckt. 1990;18:96-7.

10. Murata $H$, Matsumura $R$, Koyama A, et al. T cell receptor repertoire of $T$ cells in the kidneys of patients with lupus nephritis. Arthritis Rheum. 2002;46:2141-7.

11. Kita $Y$, Kuroda K, Mimori T, et al. T cell receptor clonotypes in skin lesions from patients with systemic lupus erythematosus. J Invest Dermatol. 1998;110:41-6.

12. Miles S, Isenberg DA. A review of serological abnormalities in relatives of SLE patients. Lupus. 1993;2:145-50.
13. Sakane $T$, Murakawa $Y$, Suzuki N, et al. Familial occurrence of impaired interleukin-2 activity and increased peripheral blood B cells actively secreting immunoglobulins in systemic lupus erythematosus. Am J Med. 1989;86:385-90.

14. Clark J, Bourne T, Salaman MR, et al. B Lymphocyte hiperactivity in families of patients with systemic lupus erythematosus. J Autoimmun. 1996;9:59-65.

15. Llorente L, Richaud-Patin Y, Couderc J, et al. Dysregulation of interleukin-10 production in relatives of patients with systemic lupus erythematosus. Arthritis Rheum. 1997;40:1429-35.

16. Tan EM, Cohen AS, Fries JF, et al. The 1982 revised criteria for the classification of systemic lupus erythematosus. Arthritis Rheum. 1982:25:1271-7.

17. Morice WG, Kimlinger T, Katzmann JA et al. Flow cytometric assessment of TCR-V beta expression in the evaluation of peripheral blood involvement by T-cell lymphoproliferative disorders: a comparison with conventional T-cell immunophenotyping and molecular genetic techniques. Am J Clin Pathol. 2004;121:373-83.

18. van den Beemd R, Boor PPC, van Lochem EG, et al. Flow cytometric analysis of the $V \circledast$ repertoire in healthy controls. Cytometry. 2000;40:336-45.

19. Luo W, Ma L, Wen $Q$, et al. Analysis of the interindividual conservation of $T$ cell receptor $\alpha$ - and $\beta$-chain variable regions gene in the peripheral blood of patients with systemic lupus erythematosus. Clin Exp Immunol. 2008;154:316-24.

20. Kolowos W, Gaipl US, Voll RE, et al. CD4 positive peripheral T cell from patients with systemic lupus erythematosus (SLE) are clonally expanded. Lupus. 2001;10:321-31.

21. Maynard J, Petersson K, Wilson $\mathrm{DH}$, et al. Structure of an autoimmune $\mathrm{T}$-cell receptor complexed with class II peptide-MHC: insights into MHC bias and antigen specificity. Immunity. 2005;22:81-92.

22. Brickman CM, Shoenfeld Y. The mosaic of autoimmunity. Scan J Clin Lab Suppl. 2001;235:3-15.

23. Andreassen $\mathrm{K}$, Bendiksen $\mathrm{S}$, Kjeldsen $\mathrm{E}$, et al. T cell autoimmunity to histones and nucleosomes is a latent property of the normal immune system. Arthritis Rheum. 2002;46:1270-81.

24. Desai-Mehta A, Mao C, Rajagopalan S, et al. Structure and specificity of $T$ cell receptors expresed by potentially pathogenic anti-DNA autoantibody-inducing T cells in human lupus. J Clin Invest. 1995;95:531-41.

25. Mato T, Masuko K, Misaki Y, et al. Correlation of clonal T cell expansion with disease activity in systemic lupus erythematosus. Int Immunol. 1997;9:547-54. 